This item was submitted to Loughborough's Research Repository by the author.

Items in Figshare are protected by copyright, with all rights reserved, unless otherwise indicated.

\title{
Planned aerobic exercise increases energy intake at the preceding meal
}

PLEASE CITE THE PUBLISHED VERSION

https://doi.org/10.1249/mss.0000000000002199

PUBLISHER

Lippincott, Williams \& Wilkins

\section{VERSION}

AM (Accepted Manuscript)

\section{PUBLISHER STATEMENT}

This is a non-final version of an article published in final form in Medicine \& Science in Sports \& Exercise, doi:10.1249/mss.0000000000002199.

\section{LICENCE}

CC BY-NC-ND 4.0

\section{REPOSITORY RECORD}

Barutcu, Asya, Shelley Taylor, Chris McLeod, Gemma Witcomb, and Lewis James. 2019. "Planned Aerobic Exercise Increases Energy Intake at the Preceding Meal". Loughborough University. https://hdl.handle.net/2134/11295764.v1. 
1 Planned aerobic exercise increases energy intake at the preceding meal

2

3 Asya Barutcu $^{1}$, Shelley Taylor ${ }^{1}$, Chris J. McLeod ${ }^{1}$, Gemma L. Witcomb ${ }^{1}$ and Lewis J. James ${ }^{1}$

$4{ }^{1}$ School of Sport, Exercise and Health Sciences, Loughborough University, Leicestershire, UK, 5 LE11 3TU.

6

7 Corresponding author

8 Lewis J. James

9 L.James@lboro.ac.uk

10 School of Sport, Exercise and Health Sciences

11 Loughborough University

12

13

14

15

16

17

18

19

20

21

22

23

24 


\section{Abstract}

26 Purpose: Effects of exercise on subsequent energy intake are well documented, but whether 27 pre-exercise energy intake is affected by future planned exercise is unknown. This study 28 investigated the effect of planned late-afternoon exercise on appetite and energy intake before 29 (breakfast and lunch) and after (evening meal/snacks) exercise. Methods: Twenty healthy, active participants $\left(10\right.$ male; age $23 \pm 5 \mathrm{y}$, BMI $23.7 \pm 3.2 \mathrm{~kg} / \mathrm{m}^{2}, \mathrm{VO}_{2}$ peak $44.1 \pm 5.4$ $\mathrm{ml} / \mathrm{kg} / \mathrm{min}$ ) completed randomised, counterbalanced exercise (EX) and resting (REST) trials. After trial notification, participants were provided ad libitum breakfast $(0800 \mathrm{~h})$ and lunch $(1200 \mathrm{~h})$ in the laboratory, before completing 1-h exercise (30 min cycling, 30 min running) at $75-80 \%$ maximal HR $(\mathrm{EX} ; 2661 \pm 783 \mathrm{~kJ})$ or 1 -h supine rest $(\mathrm{REST} ; 310 \pm 58 \mathrm{~kJ})$ 3-h postlunch. Participants were provided a food pack (pasta meal/snacks) for consumption postexercise (outside laboratory). Appetite was measured regularly and meal and 24-hour energy intake quantified. Results: Ad-libitum energy intake was greater during EX at lunch (EX 3450 $\pm 1049 \mathrm{~kJ}$; REST $3103 \pm 927 \mathrm{~kJ} ; P=0.004)$, but similar between trials at breakfast (EX $2656 \pm$ $1291 \mathrm{~kJ}$; REST $2484 \pm 1156 \mathrm{~kJ} ; P=0.648)$ and dinner $($ EX $6249 \pm 2216 \mathrm{~kJ}$; REST $6240 \pm 2585$ $\mathrm{kJ} ; P=0.784)$. Total 24 -hour energy intake was similar between trials $(P=0.388)$, meaning relative energy intake (24-h energy intake minus EX/REST energy expenditure), was reduced during EX (EX $9694 \pm 3313$ kJ; REST $11517 \pm 4023$ kJ; P=0.004). Conclusion: Energy intake appears to be increased in anticipation of, rather than in response to, aerobic exercise, but the increase was insufficient to compensate for energy expened during exercise, meaning aerobic exercise reduced energy balance relative to rest.

48 Key words: Appetite; energy intake; eating behavior; weight loss; exercise 
50 Obesity remains a major public health concern responsible for many deaths each year, with the

51 prevalence of overweight and obesity continuing to rise both in the UK (1) and globally (2).

52 Overweight and obesity develop due to an accumulation of body fat caused by a long-term 53 positive energy balance (i.e. energy intake greater than energy expenditure; 3). Whilst 54 conceptually simple, the mechanisms responsible for regulating energy balance are complex, 55 making treatment of overweight/obesity extremely difficult (4). Whilst there is a clear need to 56 identify strategies that help to facilitate weight loss, increases in overweight/obesity prevalence 57 must, at least partially, be caused by previously lean individuals gaining weight (5). Therefore, 58 whilst most research tends to focus on weight loss (i.e. treatment), far more research is warranted on how to maintain weight in lean individuals (i.e. prevention). Therefore, it is of interest to better understand the mechanisms by which energy balance is regulated, and affected 61 by exercise, in lean individuals.

62 To effectively attenuate energy balance, strategies that decrease energy intake and/or increase 63 energy expenditure, without compensatory alterations in the other components of energy 64 balance are warranted. Regular exercise, which increases energy expenditure, has been identified as one such strategy that may assist in the battle against obesity (4). Aerobic exercise causes effects on gut-derived endocrine mediators of appetite/energy intake, producing

67 reductions in the orexigenic hormone ghrelin and increases in the anorexigenic hormone 68 peptide tyrosine tyrosine (PYY; 6,7). Presumably due to alterations in these homeostatic 69 regulators of appetite, previous studies documenting the acute effects of exercise on appetite 70 and energy intake have typically examined energy intake in response to, rather than in 71 anticipation of, exercise. A meta-analysis of this now substantial body of evidence concluded 72 that acute exercise training does not alter energy intake in the hours after exercise compared to 
73 a resting control condition (8). Consequently, relative energy intake (energy consumed minus

74 energy expended through exercise/rest) is reduced, and an acute energy deficit is created (9).

75 Whilst chronic aerobic exercise training facilitates weight loss, studies do not report the 76 expected reduction in body mass/fat predicted from the acute responses (10-13). What

77 accounts for this less than anticipated weight loss has not been elucidated, but compensatory 78 increases in hunger and energy intake $(13,14)$, and/or decreases in non-exercise physical 79 activity (15) have been postulated. However, resting metabolic rate $(13,16,17)$ and nonexercise physical activity energy expenditure appear to be unaffected by aerobic exercise 81 training $(13,18)$. Therefore, it seems likely that compensatory increases in energy intake are 82 more likely to explain the less than expected weight loss observed with long-term aerobic 83 exercise training $(11,13,18)$. Indeed, a recent study $(13)$ reported that 12 weeks aerobic exercise training ( 5 x $500 \mathrm{kcal}$ exercise per week) produced a less than anticipated decrease in body mass/fat, which was accompanied by an increase in ad-libitum energy intake, but no change in resting metabolic rate or non-exercise physical activity.

87 Energy intake is regulated by a host of homeostatic and non-homeostatic mechanisms that 88 ultimately drive behaviour (19). Whilst exercise induces acute changes in the endocrine 89 regulators of appetite, these changes do not appear to manifest in differences in subsequent energy intake. Given exercise sessions are rarely spontaneous, there will usually be ample time

91 for an exerciser to alter their energy/nutrient intake in anticipation of exercise. Energy/nutrient 92 intake before exercise is commonly reported to increase exercise capabilities and thus 93 exercisers may, over time, upregulate energy intake in the pre-exercise period to effectively 94 prepare for the exercise session. Indeed, one recent study (20) reported that inactive overweight males who were restrained eaters chose more snack foods when they were served before exercise compared to a no exercise control trial. However, the extent to which these effects are 
97 apparent over longer periods of time, or at complete meals in proximity to exercise, is currently 98 unknown.

99 Therefore, this study aimed to investigate the effect of a planned late afternoon exercise session 100 on appetite and energy intake both before (at breakfast and lunch) and after (evening $101 \mathrm{meal} / \mathrm{snacks}$ ) exercise and to compare these responses to an identical resting control trial. It 102 was hypothesised that energy intake at breakfast and lunch, but not in the evening after 103 exercise, would be greater for exercise compared to rest.

104 


\section{Methods}

107 Participants were twenty healthy, non-smoking, weight stable (self-reported), habitually active 108 ( $<10$ hours per week) males $\left(\mathrm{n}=10\right.$; age $23 \pm 6$ years; BMI $23.9 \pm 3.3 \mathrm{~kg} / \mathrm{m}^{2}$; body fat $16.3 \pm$ $\left.4.2 \% ; \mathrm{VO}_{2} \max 47.7 \pm 4.0 \mathrm{ml} / \mathrm{kg} / \mathrm{min}^{-1}\right)$ and females $(\mathrm{n}=10$; age $24 \pm 4$ years; $\mathrm{BMI} 23.5 \pm 3.2$ $110 \mathrm{~kg} / \mathrm{m}^{2}$; body fat $\left.28.6 \pm 6.3 \% ; \mathrm{VO}_{2} \max 40.6 \pm 4.3 \mathrm{ml} / \mathrm{kg} / \mathrm{min}^{-1}\right)$. participants provided written 111 consent before taking part in the study. Ethical approval was obtained from the Loughborough

112 University Ethics Approvals (Human Participants) Sub Committee (reference number: R17P024). Participants were not taking any medications known to affect appetite, and they were

114 also not restricted, disinhibited, or hungry eaters, as determined by the Three-Factor Eating 115 Questionnaire (21). Each participant completed two preliminary trials and two experimental 116 trials in a randomised counterbalanced order and separated by 4-14 days. All females were 117 using the combined oral contraceptive pill, with all trials taking place after at least 3 days of 118 continuous contraceptive pill use. In the absence of any data to inform the size of the anticipated 119 effect, the sample size used was in line with previous studies in this area using a similar cross120 over design.

\section{Pre-trial standardisation}

122 In the $24 \mathrm{~h}$ preceding the first experimental trial, participants recorded their dietary intake and 123 habitual physical activity. These diet and activity patterns were then replicated prior to the 124 second experimental trial. Strenuous exercise and alcohol intake were not permitted during this $12524 \mathrm{~h}$ pre-trial period and adherence to all pre-trial requirements were verbally checked before 126 trials. 


\section{Preliminary trials}

129 During the first preliminary trial, height (to nearest $0.1 \mathrm{~cm}$; SECA stadiometer, Germany) and 130 body mass (to nearest $0.01 \mathrm{~kg}$; Adam Equipment, CFM-150 scales, UK) were measured, whilst 131 body composition was estimated using skinfold thickness (Harpenden, UK) at four sites 132 (biceps, triceps, sub-scapula, supra-iliac; 22). Participants then completed questionnaires to assess health status and eating patterns, before performing two submaximal exercise tests; one on a cycle ergometer (Lode Corival, Groningen, Holland) and one on a treadmill ( $\mathrm{h} / \mathrm{p} / \mathrm{cosmos}$ sports \& medical gmbh, Germany). These submaximal tests involved four incremental 4-min stages on both a cycle ergometer (at workloads between $80-280 \mathrm{~W}$ ) and treadmill (at speeds

137 between 6-13 km/h), with the specific intensities used dependent on each participant's fitness.

138 Heart rate (Polar M400, Kempele, Finland) and rating of perceived exertion (RPE; 23) were 139 recorded at the end of each 4-min stage.

140 After a short break, participants completed a maximal incremental exercise test on the treadmill

141 to determine their peak oxygen uptake $\left(\mathrm{VO}_{2}\right.$ peak $)$. Exercise started at a gradient of $1 \%$ and at 142 a speed estimated to elicit a heart rate of $\sim 160$ beats/min, with the gradient increasing by $1 \%$ 143 every min until volitional exhaustion. Expired gas was collected during the final min of the 144 maximal incremental exercise test, with heart rate and RPE recorded at the end of each 1-min 145 increment. During the second preliminary trial, participants arrived at the laboratory at $0800 \mathrm{~h}$ 146 in a fasted state and completed visual analogue scales to assess subjective appetite, consisting 147 of ratings of hunger, fullness, desire to eat (DTE) and prospective food consumption (PFC).

148 After 25 min supine rest, a 5-min expired gas sample was collected into a Douglas Bag to 149 determine resting energy expenditure. Participants were then familiarized with experimental procedures by replicating procedures described below for the exercise trial, including appetite 
151 questionnaires, ad-libitum breakfast and lunch meals, the exercise session and the ad-libitum 152 evening food intake.

\section{Experimental trials}

154 Participants completed two experimental trials; exercise (EX) and rest (REST) in a randomised counter-balanced order and separated by at least 4 days. Participants arrived at the laboratory at $0800 \mathrm{~h}$ in a fasted state and baseline measures of subjective appetite and post-void body mass

157 in light clothing were made $(0800$ h). Participants were then informed if they were on the EX 158 or REST trial that day, before subjective appetite was again measured 15 min later $(0815 \mathrm{~h})$. 159 Participants were then given 30 min to consume breakfast, which consisted of a multi-item cold-food buffet, with subjective appetite measured again post-breakfast (0845 h). Before eating breakfast, participants were provided the following standard instructions "You have 30

162 min to eat your breakfast. Remember that you are on the exercise/rest trial today, so please

163 choose your food items accordingly. You are welcome to eat whatever and how much you want 164 from the selection. If you want more of anything, please let us know and we will put out more food." Participants left the laboratory after breakfast and continued with their daily activities (restricted to low-intensity activities), returning for lunch at $1200 \mathrm{~h}$, which again consisted of

167 a multi-item cold-food buffet for a period of $30 \mathrm{~min}$. Before lunch, participants were given the 168 same trial-specific instructions as before breakfast. Subjective appetite was measured before 169 (1200 h) and after (1230 h) lunch. Participants then rested quietly in the laboratory for the next $1703 \mathrm{~h}$, with subjective appetite measured every hour (1330 h, $1430 \mathrm{~h}, 1530 \mathrm{~h})$, before they 171 completed the exercise/rest session. In the EX trial, exercise consisted of $30 \mathrm{~min}$ of steady state 172 cycling at $75 \%$ heart rate-max, followed by 30 min of steady state running at $80 \%$ heart rate173 max. Heart rate and RPE were recorded every 5 min throughout exercise. Expired gas samples 174 were collected between 14-15 min and 29-30 min during cycling and running. In the REST 
175 trial, participants completed the equivalent duration of supine rest, with expired gas samples 176 collected between 25-30 min and 55-60 min. Subjective appetite was measured at $30 \mathrm{~min}$ (1600

177 h) and upon completion (1630 h) of the exercise/rest period. Participants were then provided a 178 food pack (main meal and snack options) to eat from over the evening and were free to leave 179 the laboratory. Participants were also given appetite questionnaires to complete at certain times 180 outside the laboratory (pre-evening meal, post-evening meal, before bed, morning).

\section{$181 \quad$ Study Foods}

182 Participants were only permitted to eat foods provided to them during experimental trials but 183 were free to drink water ad-libitum throughout trials (including during the exercise/rest 184 periods). For all meals, food was provided in excess of expected consumption. For breakfast and lunch meals only, additional food was available on request. Foods provided at breakfast,

186 lunch and evening are presented in Table 1. For breakfast and lunch meals, foods were 187 presented in a research kitchen, where participants were able to serve and/or make food items, 188 before moving to a separate dining room to eat. For these meals, participants ate in isolation and there was no interaction between researcher and participants, with participants free to select

190 foods they wanted. For the evening food pack, participants were provided with a main meal 191 (cheese and tomato pasta), along with a standard bowl, and a variety of snacks. Participants 192 were instructed to bring back any leftover items (including wrapping and fruit skins) for 193 accurate measurements of energy intake and told that they were free to keep any food items 194 after the food pack was re-measured. The pasta meal was prepared on the day of the 195 experimental trial using standard cooking and cooling procedures and was given to participants 196 cold. The cheese and tomato pasta provided $6.63( \pm 0.03 \mathrm{SD}) \mathrm{kJ} \cdot \mathrm{g}^{-1}$ (with $14 \%, 60 \%, 25 \%$ and $1971 \%$ of the energy provided by protein, carbohydrate, fat and fibre, respectively). 
198 Participants completed questionnaires related to liking of study foods to ensure the available

199 foods were adequately palatable. For each meal, food consumed was quantified by weighing 200 foods before and after consumption and taking into account any leftovers. Energy and 201 macronutrient content of foods was ascertained from manufacturer values. Upon arrival for 202 lunch, participants verbally confirmed that they had not eaten/drunk anything except water 203 since breakfast and upon returning uneaten evening food, that they had only eaten food items 204 from the food pack

\section{Subjective Appetite Sensations}

206 Using paper and pen scales, participants rated their feelings of hunger 'How hungry do you 207 feel?', fullness 'How full do you feel?', desire to eat (DTE) 'How strong is your desire to eat?', 208 and prospective food consumption (PFC) 'How much food do you think you could eat?' on $209100 \mathrm{~mm}$ visual analogue scales throughout the day. Verbal anchors of "not at all/none at all/no 210 desire at all" and "extremely/a lot" were placed at 0 and $100 \mathrm{~mm}$, respectively.

\section{$211 \quad$ Statistical Analysis}

212 Data were analysed using SPSS 23.0 (SPSS Inc., Somers, NY, USA). All data were checked 213 for normality of distribution using a Shapiro-Wilk test. Sex differences were initially 214 explored through two-way (sex*trial) or three-way (sex*trial*time) repeated measures 215 ANOVA. Where interaction effects were observed (energy expenditure during the $1 \mathrm{~h}$ 216 exercise/rest and fullness), data were analysed with sexes separated and combined. All other 217 data were analysed for both sexes combined. Significant interaction effects were followed by

218 Bonferroni-adjusted paired t-tests or Bonferroni-adjusted Wilcoxon signed-rank tests, as 219 appropriate. Data containing one factor were analysed using a t-test or Wilcoxon signed-rank 220 test, as appropriate. Data sets were determined to be significantly different when $\mathrm{P}<0.05$.

221 Data are presented as mean \pm standard deviation throughout, unless otherwise stated. 


\section{Results}

223

22

225

226

227

228

229

230

231

232

233

234

235

236

237

238

239

240

241

242

243

244

\section{Pre-trial measures}

There were no differences between trials for pre-trial body mass $(\mathrm{t}=-1.243 ; P=0.229)$, or subjective appetite sensations of hunger $(\mathrm{Z}=-0.318 ; P=0.763)$, fullness $(\mathrm{Z}=-0.201 ; P=$ 0.852), DTE $(\mathrm{Z}=-0.486 ; P=0.641)$ and PFC $(\mathrm{Z}=-1.007 ; P=0.327)$.

\section{Energy and Macronutrient Intake}

Energy intake at the different eating occasions and over the $24 \mathrm{~h}$ is presented in Table 2 . Energy intake at breakfast $(\mathrm{Z}=-0.485 ; P=0.648)$ and during the evening $(\mathrm{Z}=-0.299 ; P=0.784)$ were similar between trials, but lunch energy intake was increased by $~ 11 \%$ in EX compared to REST $(\mathrm{t}=3.324 ; P=0.004)$. Furthermore, total pre-exercise/rest energy intake (breakfast + lunch) was $\sim 9 \%$ greater in EX compared to REST $(\mathrm{t}=2.212 ; P=0.039)$. However, total $24 \mathrm{~h}$ energy intake was similar between trials $(\mathrm{Z}=-0.896 ; P=0.388)$. Relative energy intake (total $24 \mathrm{~h}$ energy intake minus energy expended through exercise/rest) was reduced by $\sim 16 \%$ in EX compared to REST (EX $9694 \pm 3313 \mathrm{~kJ}$; REST $11517 \pm 4023 \mathrm{~kJ} ; \mathrm{Z}=-2.800 ; P=0.004$ ).

There were no differences between trials for carbohydrate, fat, protein and fibre intakes $(P \geq$ $0.245)$ at breakfast or over the evening (Table 2). However, protein $(\mathrm{t}=2.657 ; P=0.016)$ and fat $(\mathrm{t}=3.369 ; P=0.003)$ intakes at lunch were greater in EX compared to REST, with carbohydrate and fibre intake at lunch similar between trials $(P \geq 0.059)$.

There were no sex*trial interaction effects for energy intake at breakfast $(F(1)=0.061 ; P=$ 0.808), lunch $(F(1)=0.018 ; P=0.893)$, breakfast + lunch $(F(1)=0.019 ; P=0.893)$, in the evening $(F(1)=1.218 ; P=0.284)$ or over the $24 \mathrm{~h}(F(1)=0.702 ; P=0.413)$. There was a sex*trial interaction effect for energy expenditure during the $1 \mathrm{~h}$ exercise/rest $(F(1)=22.835$; $P<0.001$ ), with the energy expended during exercise representing a greater proportion of 
245 energy expenditure during the $1 \mathrm{~h}$ rest in males (Male $939 \pm 164 \%$; female $776 \pm 142 \%$; $P=$ 246 0.028). Consequently, there was a trend for a sex*trial interaction for relative energy intake $247 \quad(F(1)=3.660 ; P=0.072)$.

\section{Subjective appetite sensations}

249 There were time and trial*time interaction effects for all subjective appetite ratings (Figure 2; $250 P<0.05)$. Additionally, there were trial effects for hunger $(F(1)=4.611 ; P=0.045), \mathrm{DTE}(F(1)$ $251=4.741 ; P=0.042)$ and PFC $(F(1)=10.251 ; P=0.005)$, but not fullness $(F(1)=0.352 ; P=$ 252 0.560). participants reported lower hunger, PFC and DTE at $1600 \mathrm{~h}$ and $1630 \mathrm{~h}$ (i.e. mid253 exercise and post-exercise, respectively) in EX $(P<0.05)$, with DTE also reduced at $1530 \mathrm{~h}$ 254 (i.e. pre-exercise). PFC was lower, and fullness was higher at $1230 \mathrm{~h}$ (i.e. immediately post255 lunch) in EX vs REST $(P<0.01)$, with fullness lower after the evening meal in EX vs REST $256(P<0.05)$.

257 There was a trial*time*sex interaction effect for fullness $(F(6.095)=2.315 ; P=0.038)$, with 258 the only significant post-hoc difference within or between sex, being that males reported greater 259 fullness at $1230 \mathrm{~h}$ (i.e. post-lunch) in EX vs REST (EX $87 \pm 7 \mathrm{~mm}$; REST $79 \pm 9 \mathrm{~mm} ; t=$ $260 \quad 5.622 ; P=0.005)$.

\section{Steady state exercise and energy expenditure}

262 Mean RPE and heart rate during the 60-min exercise in EX were $12 \pm 1$ and $147 \pm 19$ bpm, 263 respectively. Mean $\mathrm{RER}, \mathrm{VO}_{2}$, carbohydrate and fat oxidation over the $60 \mathrm{~min}$ exercise/rest 264 were all greater during EX compared to REST $(P<0.001$; Table 3$)$. 
268 This study investigated the effect of a planned 60-min late-afternoon aerobic exercise session on appetite and energy intake both before (i.e. at breakfast and lunch) and after (i.e. over the evening) exercise compared to an identical resting control trial. It was hypothesised that energy

271 intake before exercise (i.e. at breakfast and lunch) would be greater than before rest, but that 272 energy intake in the evening would be similar between trials. In line with this hypothesis, 273 energy intake in the pre-exercise/pre-rest period was significantly greater $(\sim 9 \%)$ in the EX trial, 274 whilst energy intake over the evening was similar between trials. Interestingly, the increased 275 energy intake before exercise was mainly caused by an $\sim 11 \%$ increase in energy intake at lunch, 276 whilst energy intake at breakfast was not different between trials.

277 To our knowledge, this is the first study to investigate energy intake and appetite responses at 278 meals consumed both before and after a planned exercise session, compared to a resting control trial. Previous studies examining the acute effects of exercise on energy intake have generally employed the approach of assessing appetite and energy intake following exercise/rest $(6,24,25)$. Aerobic exercise has been shown to modulate circulating concentrations of acylated ghrelin and PYY, hormones secreted from the gatrointestinal tract that are thought to play a role in the regulation of appetite and energy intake (26,27). Interestingly, and perhaps counterintuitively, aerobic exercise decreases acylated ghrelin concentrations and increases PYY concentrations, producing a hormonal melieu conducive to the suppression of appetite/energy intake (7). Despite these consistent effects on hormonal mediators of appetite, acute exercise studies mainly suggest that energy intake after exercise is no different to after a similar duration of rest (8). Therefore, relative energy intake (energy intake minus energy expended through exercise/rest) is reduced with aerobic exercise, suggesting exercise helps to facilitate an acute negative energy balance. The present study supports the findings of these 291 previous studies as energy intake after exercise was similar between EX and REST trials, but 292 demonstrates that regular exercisers might increase their energy intake in anticipation of an 
exercise session. However, this increase in pre-exercise energy intake was not sufficient to

294 offset the extra energy expended during exercise, meaning that exercise reduced relative energy

295 intake compared to the rest trial.

296 In a similar recent study, Sim, Lee and Cheon (20) investigated the effects of a future exercise 297 bout on pre-exercise energy intake in inactive overweight males. After standardised breakfast 298 and lunch meals, participants were provided an ad-libitum snack (potato chips) an hour before 299 a known exercise (self-selected exercise duration/intensity) or a rest session. Whilst overall 300 there was no effect of exercise on energy intake, the authors observed that restrained eaters ate 301 significantly more ( $\sim 162 \mathrm{kcal}$ or $\sim 677 \mathrm{~kJ})$ before exercise, an effect that was not present in the 302 unrestrained eaters. In contrast to the results of Sim et al. (20), the present study observed that 303 unrestrained eaters increased their energy intake at a pre-exercise meal in anticipation of a $1 \mathrm{~h}$ aerobic exercise session. There are a number of differences in study design that likely account for these discordant findings. Firstly, in the present study, participants were provided with two multi-item buffet meals (breakfast and lunch) $7.5 \mathrm{~h}$ and $3.5 \mathrm{~h}$ before exercise, respectively,

307 whilst in the study of Sim et al. (20) participants were provided only a pre-exercise ad-libitum 308 snack of potato chips $1 \mathrm{~h}$ before exercise. The additional opportunities to eat, choice of foods or the more distal (but more realistic) positioning of meals relative to exercise in the present 310 study might have provided greater opportunity to increase energy intake in the exercise trial. 311 Furthermore, participants in the present study were regular exercisers, whereas those in the 312 study of Sim et al. (20) were inactive individuals. The lack of experience with exercise of the 313 participants in this previous study (20), compared to participants in the present study, may have 314 reduced their propensity to increase energy intake in anticipation of exercise. Alternatively, the 315 fact that participants in the present study were not attempting to lose weight might mean that 316 they were more likely to increase their energy intake in anticipation of exercise (although 
317 exercise still created an energy deficit). Future studies should look to examine these effects in

318 those attempting to lose weight, who might be less likely to increase energy intake.

319 Previous work has demonstrated that there are elements of eating behaviour that are learned,

320 with experience of a food influencing expectations about a food's satiation (28). Indeed, 321 expected satiety/satiation are strong predictors of portion size selection $(29,30)$. Although speculative, it might be hypothesised that exercise (or energy expenditure per se) might illicit

323 a similar response, where previous experience with an exercise task might facilitate learned 324 increases in portion size selection and energy intake. In line with this hypothesis, Werle et al. 325 (31) observed that energy served from snacks was increased in participants who answered a 326 series of questions related to exercise, compared to those that answered questions unrelated to exercise (31). Thus in the present study, participants' previous experience with aerobic exercise might have meant they had 'learned' to increase their energy intake in the pre-exercise period 329 to prepare for the coming exercise/energy expenditure. Whilst speculative, this hypothesis 330 might go some way to explain the results of chronic training studies, where weight loss slows 331 down over time $(11,32)$. Alternatively, it is possible that the pre-execise period represents a 332 time where exercisers are more likely to increase energy intake to compensate for impending 333 energy expenditure. Indeed, in support of this theory, a recent study (33) observed that planned 334 energy intake at a future lunch meal was increased when participants were told the meal would 335 be consumed after $1 \mathrm{~h}$ of hard aerobic exercise compared after a period of rest.

336 Alternatively, the results of the present study might be explained by other possible mechanisms. 337 Firstly, the Compensatory Health Beliefs Model (34) postulates that certain unhealthy 338 behaviours can be compensated for by positive (healthy) behaviours, and this model might, at 339 least partially, explain the findings. Knowledge of a planned future exercise session (perceived 340 as a healthy behaviour) might allow an exerciser to justify, to themselves, having extra 
341 energy/food (perceived as an unhealthy behaviour) in the lead up to exercise $(35,36)$. Secondly,

342 and on a similar line to the health beliefs model, general scientific recommendations are for

343 athletes to increase energy, and particularly carbohydrate, intake in the hours before exercise

344 (37). As these recommendations, which are made for athletes, permeate into lay

345 publications/online resources, they might promulgate the idea that exercisers (not only athletes)

346 should increase their food and energy intake to appropriately prepare for a future exercise

347 session. Interestingly, there was no difference in pre-exercise carbohydrate intake, although

348 energy, protein and fat intake where all higher in the EX trial. However, an increase in energy

349 (or indeed carbohydrate) intake after exercise would also be predicted by the compensatory

350 health beliefs model and would also be consistent with current scientific recommendations for

351 athletes (37), but this was not found. Therefore, the finding that energy and macronutrient

352 intakes in the evening were similar between REST/EX trials suggests that these possible

353 mechanisms are not likely to explain the findings. One consideration is the wording used to

354 inform participants of which trial they are on. We aimed to ensure participants had the

355 impending exercise/rest in mind when making decisions about food to consume, although this

356 meant that the wording was possibly leading. Whilst this possibly represents a limitation of the

357 present work, the fact that an increased energy intake was observed at lunch, but not at

358 breakfast, suggests that the wording did not bias participants to eat more food (energy) in the

359 exercise trial. That said, given this is one of the first studies to investigate these effects, future

360 studies should carefully consider how information about the impending exercise sessions is

361 given to participants.

362 Whilst the mechanism explaining the present results remains to be elucidated, the findings 363 suggest that energy intake is increased in anticipation of, rather than in response to, exercise.

364 These findings for post-exercise energy intake are similar to those reported in the vast majority 365 of the previous literature in this area (8). Although energy intake was significantly increased 
before exercise, the increase was only $\sim 518 \mathrm{~kJ}(\sim 124 \mathrm{kcal})$ and when this was combined with

367 the energy intake post-exercise, there was no significant difference between trials, although mean energy intake was arithmetically greater in the EX trial. Furthermore, when the energy expended during the $60 \mathrm{~min}$ exercise/rest was factored in, relative energy intake was $\sim 1823 \mathrm{~kJ}$

370 ( $\sim 36 \mathrm{kcal})$ less in the EX trial. In this regard, the present study is consistent with the vast

371 majority of the previous literature examining the short-term effects of exercise on ad-libitum energy intake $(6,8,24-26)$. The present study, along with these previous studies, demonstrates

373 that a single bout of aerobic exercise does not induce a substantial increase in energy intake

374 around exercise, thus facilitating an energy deficit that should be conducive to weight loss if 375 exercise training continues. The present study only explored the period immediately preceding 376 an exercise session and given that exercise sessions are generally planned well in advance (i.e. an exerciser might habitually do exercise classes on a particular day of the week every week),

378 there may be further opportunity to increase energy intake prior to exercise. Future studies

379 should examine eating behaviour over longer periods before exercise, as well as how eating 380 behaviour before and after exercise is affected by long-term exercise training. Furthermore, 381 whether diet goals (i.e. maintain vs lose weight) influence these responses should also be 382 investigated.

In conclusion, this study demonstrates that energy intake is increased in anticipation of aerobic

384 exercise (i.e. before exercise), rather than in response to the exercise session (i.e. after exercise).

385 The increase in energy intake was not sufficient to offset the energy deficit created by the exercise session, meaning that aerobic exercise reduced energy balance relative to rest, which is consistent with previous literature examining post-exercise energy intake. However, the 388 finding that energy intake is increased in anticipation of an aerobic exercise session perhaps 389 changes our understanding of how exercise might influence energy intake and, speculatively, 390 suggests regular exercisers might 'learn' to increase their energy intake in preparation for an 
391 exercise session, a behaviour that might attenuate the negative energy balance induced by exercise. However, clearly further research is needed to better understand this phenomonen.

393

394 Acknowledgements

395 LJJ is part of the National Institute for Health Research (NIHR) Leicester Biomedical Research 396 Centre, which is a partnership between University Hospitals of Leicester NHS Trust, 397 Loughborough University and the University of Leicester. This report is independent research 398 by the National Institute for Health Research. The views expressed in this publication are those 399 of the authors and not necessarily those of the NHS, the National Institute for Health Research 400 or the Department of Health. No other funding was received for this study.

401 The authors declare no conflict of interest.

402 The results of the present study do not constitute endorsement by the American College of 403 Sports Medicine. 


\section{References}

405 1. Conolly A, Saunders C. Health Survey for England: 2016: Adult overweight and $406 \quad$ obesity. NHS Digit. 2017;1(December):1-21.

407 2. Abarca-Gómez L, Abdeen ZA, Hamid ZA, Abu-Rmeileh NM, Acosta-Cazares B, 408 Acuin C, et al. Worldwide trends in body-mass index, underweight, overweight, and 409 obesity from 1975 to 2016: a pooled analysis of 2416 population-based measurement

412 3. Schrauwen P. High-fat diet, muscular lipotoxicity and insulin resistance. Proc Nutr Soc. 2007;66(1):33-41.

414 4. Lean MEJ, Astrup A, Roberts SB. Making progress on the global crisis of obesity and $415 \quad$ weight management. BMJ. 2018;361.

416 5. Ostbye T, Malhotra R, Landerman LR. Body mass trajectories through adulthood: results from the National Longitudinal Survey of Youth Cohort (1981-2006). Int J Epidemiol 2011;40(1):240-50.

419 6. King JA, Miyashita M, Wasse LK, Stensel DJ. Influence of prolonged treadmill running on appetite, energy intake and circulating concentrations of acylated ghrelin. Appetite. 2010;54(3):492-8.

422 7. Dorling J, Broom DR, Burns SF, Clayton DJ, Deighton K, James LJ, et al. Acute and chronic effects of exercise on appetite, energy intake, and appetite-related hormones: The modulating effect of adiposity, sex, and habitual physical activity. Nutrients.

425 $2018 ; 10(9)$.

426 8. Schubert MM, Desbrow B, Sabapathy S, Leveritt M. Acute exercise and subsequent 
energy intake. A meta-analysis. Appetite. 2013;63:92-104.

428 9. Caudwell P, Gibbons C, Hopkins M, Naslund E, King N, Finlayson G, et al. The influence of physical activity on appetite control: An experimental system to understand the relationship between exercise-induced energy expenditure and energy intake. Proc Nutr Soc. 2011;70(2):171-80.

10. Wu T, Gao X, Chen M, Van Dam RM. Long-term effectiveness of diet-plus-exercise interventions vs. diet-only interventions for weight loss: A meta-analysis: Obesity Management. Obes Rev. 2009;10(3):313-23.

11. Turner JE Betts JA, Thompson D MD. Non prescribed physical activity energy expenditure is maintained with structured exercise and implicates a compensatory increase in energy intake. Am J Clin Nutr. 2010;92:1009-16.

12. Rocha J, Paxman J, Dalton C, Winter E, Broom DR. Effects of a 12-week aerobic exercise intervention on eating behaviour, food cravings, and 7-day energy intake and energy expenditure in inactive men. Appl Physiol Nutr Metab. 2016;41(11):1129-36.

441 13. Myers A, Dalton M, Gibbons C, Finlayson G, Blundell J. Structured, aerobic exercise reduces fat mass and is partially compensated through energy intake but not energy expenditure in women. Physiol Behav. 2019 Feb;199:56-65.

444 14. Hopkins M, King NA, Blundell JE. Acute and long-term effects of exercise on appetite control: Is there any benefit for weight control? Curr Opin Clin Nutr Metab Care. 2010;13(6):635-40.

447 15. Kozey-Keadle S, Staudenmayer J, Libertine A, Mavilia M, Lyden K, Braun B, et al. $448 \quad$ Changes in Sedentary Time and Physical Activity in Response to an Exercise Training 449 and/or Lifestyle Intervention. J Phys Act Heal. 2014;11(7):1324-33. 
450 16. Speakman JR, Selman C. Physical activity and resting metabolic rate. Proc Nutr Soc. 2003;62(03):621-34.

452 17. Lee MG, Sedlock DA, Flynn MG, Kamimori GH. Resting metabolic rate after 453 endurance exercise training. Med Sci Sports Exerc. 2009;41(7):1444-51.

454 18. Fedewa M V., Hathaway ED, Williams TD, Schmidt MD. Effect of Exercise Training 455 on Non-Exercise Physical Activity: A Systematic Review and Meta-Analysis of Randomized Controlled Trials. Sport Med. 2017;47(6):1171-82.

19. Blundell JE. The contribution of behavioural science to nutrition: Appetite control. Nutr Bull. 2017;42(3):236-45.

20. Sim AY, Lee LL, Cheon BK. When exercise does not pay: Counterproductive effects of impending exercise on energy intake among restrained eaters. Appetite. 2018;123:120-7.

462

21. Stunkard AJ, Messick S. The three-factor eating questionnaire to measure dietary restraint, disinhibition and hunger. J Psychosom Res. 1985;29(1):71-83.

22. Durnin J V, Womersley J. and Its Estimation From Skinfold Thickness : Measurements on. Br J Nutr. 1973;32(1):77-97.

23. Borg GAV. Psychophysical bases of perceived exertion. Med Sci Sport Exerc. $1982 ; 14(5): 377-81$.

24. King JA, Wasse LK, Broom DR, Stensel DJ. Influence of brisk walking on appetite, energy intake, and plasma acylated ghrelin. Med Sci Sports Exerc. 2010;42(3):485-92.

470 25. Deighton K, Zahra JC, Stensel DJ. Appetite, energy intake and resting metabolic 471 responses to $60 \mathrm{~min}$ treadmill running performed in a fasted versus a postprandial state. 
473 26. Douglas JA, King JA, McFarlane E, Baker L, Bradley C, Crouch N, et al. Appetite, appetite hormone and energy intake responses to two consecutive days of aerobic exercise in healthy young men. Appetite. 2015;92:57-65.

27. King JA, Garnham JO, Jackson AP, Kelly BM, Xenophontos S, Nimmo MA. Appetiteregulatory hormone responses on the day following a prolonged bout of moderateintensity exercise. Physiol Behav. 2015;141:23-31.

479

480

481

482

483

484

485

486

487

488

489

490

491

492 493

29. Brunstrom JM, Rogers PJ. How many calories are on our plate expected fullness, not liking, determines meal-size selection. Obesity. 2009;17(10):1884-90.

30. Brunstrom JM, Shakeshaft NG. Measuring affective (liking) and non-affective (expected satiety) determinants of portion size and food reward. Appetite. 2009;52(1):108-14.

31. Werle COC, Wansink B, Payne CR. Just thinking about exercise makes me serve more food. Physical activity and calorie compensation. Appetite. 2011;56(2):332-5.

32. Curioni CC, Lourenço PM. Long-term weight loss after diet and exercise: A systematic review. Int J Obes. 2005;29(10):1168-74.

33. Barutcu A, Witcomb GL, James LJ. Anticipation of aerobic exercise increases planned energy intake for a post-exercise meal. Appetite. 2019;138:198-203.

34. Thongworn S, Sirisuk V. Weight control specific compensatory health beliefs: Hypothetical testing and model extension. Kasetsart J Soc Sci. 2018;39(2):312-9. 
494 35. Rabiau MA, Knäuper B, Nguyen TK, Sufrategui M, Polychronakos C. Compensatory 495 beliefs about glucose testing are associated with low adherence to treatment and poor 496 metabolic control in adolescents with type 1 diabetes. Health Educ Res.

$497 \quad 2009 ; 24(5): 890-6$.

498 36. King NA. What processes are involved in the appetite response to moderate increases in exercise-induced energy expenditure? Proc Nutr Soc. 1999;58(01):107-13.

500 37. Burke LM, Hawley JA, Wong SHS, Jeukendrup AE. Carbohydrates for training and $501 \quad$ competition. J Sports Sci. 2011;29(Supp 1):S17-S27.

502 
503 Table 1. Food items provided at meals.

\begin{tabular}{|c|c|c|}
\hline \multicolumn{3}{|c|}{ Breakfast buffet items } \\
\hline White bread & Cornflakes - cereal & Peanut butter spread \\
\hline Brown Bread & Weetabix - cereal & Nutella spread \\
\hline Rice crispies - cereal & Strawberry yoghurt & Strawberry jam spread \\
\hline Crunchy nut - cereal & Raspberry yoghurt & Banana \\
\hline Shreddies - cereal & Cherry yoghurt & Apples \\
\hline Coco pops - cereal & Apple juice & Clementine \\
\hline Cheerios - cereal & Orange juice & Milk \\
\hline \multicolumn{3}{|c|}{ Lunch buffet items } \\
\hline White bread & Cherry yoghurt & Salt and vinegar crisps \\
\hline Brown Bread & Strawberry yoghurt & Cheese and onion crisps \\
\hline Mature cheddar cheese & Raspberry yoghurt & Orange squash \\
\hline Honey smoked ham & Cadbury mini rolls & Summer fruits squash \\
\hline Grilled chicken pieces & Mayonnaise & Apples \\
\hline Can of tuna & Butter & Clementine \\
\hline Lettuce & Chocolate chip cookies & \\
\hline Tomato & Salted crisps & \\
\hline \multicolumn{3}{|c|}{ Evening meal } \\
\hline Nutrigrain apple cereal bar & Cheese and onion crisps & Clementine \\
\hline $\begin{array}{l}\text { Nutrigrain blueberry cereal } \\
\text { bar }\end{array}$ & Prawn cocktail crisps & Banana \\
\hline $\begin{array}{l}\text { Nutrigrain strawberry } \\
\text { cereal bar }\end{array}$ & Salt and vinegar crisps & Strawberry yoghurt \\
\hline Mars chocolate - fun size & Salted crisps & Cherry yoghurt \\
\hline Twix chocolate - fun size & Mini cookies & Raspberry yoghurt \\
\hline $\begin{array}{l}\text { Maltesers chocolate - fun } \\
\text { size }\end{array}$ & Apple & Tomato pasta meal \\
\hline
\end{tabular}

505 Table 2. Total energy $(\mathrm{kJ})$, carbohydrate $(\mathrm{CHO})$, protein $(\mathrm{PRO})$, fat, and fibre intake over the 506 course of each trial. 


\begin{tabular}{|c|c|c|c|c|c|}
\hline & Energy (kJ) & CHO (g) & PRO (g) & FAT (g) & Fibre (g) \\
\hline & & & Breakfast & & \\
\hline EX & $2656 \pm 1291$ & $108.5 \pm 49.9$ & $18.9 \pm 11.3$ & $12.5 \pm 9.6$ & $6.3 \pm 4.9$ \\
\hline \multirow[t]{2}{*}{ REST } & $2484 \pm 1156$ & $103.8 \pm 45.9$ & $18.3 \pm 9.5$ & $10.5 \pm 6.9$ & $5.6 \pm 4.1$ \\
\hline & & & Lunch & & \\
\hline EX & $3450 \pm 1049^{\dagger}$ & $74.3 \pm 22.9$ & $38.7 \pm 13.7^{\dagger}$ & $39.5 \pm 17.4^{\dagger}$ & $8.3 \pm 2.5$ \\
\hline \multirow[t]{2}{*}{ REST } & $3103 \pm 927$ & $70.3 \pm 20.7$ & $34.4 \pm 12.9$ & $34.2 \pm 15.2$ & $7.7 \pm 2.3$ \\
\hline & \multicolumn{5}{|c|}{ Bfast + Lunch } \\
\hline EX & $6105 \pm 1980^{\dagger}$ & $182.8 \pm 68.3$ & $57.7 \pm 21.1^{\dagger}$ & $52.0 \pm 19.7^{\dagger}$ & $14.6 \pm 7.0$ \\
\hline \multirow[t]{2}{*}{ REST } & $5588 \pm 1933$ & $174.0 \pm 64.4$ & $52.7 \pm 19.3$ & $44.6 \pm 19.0$ & $13.4 \pm 6.0$ \\
\hline & \multicolumn{5}{|c|}{ Evening Meal } \\
\hline EX & $6249 \pm 2216$ & $223.2 \pm 81.0$ & $40.1 \pm 14.1$ & $43.8 \pm 15.7$ & $10.2 \pm 4.3$ \\
\hline \multirow[t]{2}{*}{ REST } & $6240 \pm 2585$ & $229.4 \pm 100.8$ & $41.6 \pm 15.7$ & $45.7 \pm 19.2$ & $10.5 \pm 4.9$ \\
\hline & \multicolumn{5}{|c|}{ Total 24h } \\
\hline EX & $12354 \pm 3920$ & $405.9 \pm 141.7$ & $97.7 \pm 32.4$ & $95.8 \pm 27.7$ & $24.8 \pm 10.7$ \\
\hline REST & $11827 \pm 4069$ & $403.4 \pm 151.5$ & $94.3 \pm 31.9$ & $90.3 \pm 31.7$ & $23.9 \pm 9.9$ \\
\hline
\end{tabular}

508

509

510

511

512

513 
514 Table 3. Mean RER, VO2, carbohydrate and fat oxidation values for EX and REST trials.

\begin{tabular}{ccccc}
\hline & $\mathbf{V O}_{2}$ & RER & Carbohydrate oxidation & Fat Oxidation \\
& $\left.(\mathbf{l . m i n})^{-1}\right)$ & & $\left(\mathbf{g} \cdot \mathbf{m i n}^{-\mathbf{1}}\right)$ & $\left(\mathbf{g . m i n}^{-\mathbf{1}}\right)$ \\
\hline EX & $2.02 \pm 0.166^{\dagger}$ & $0.96 \pm 0.03^{\dagger}$ & $2.369 \pm 0.088^{\dagger}$ & $0.125 \pm 0.098^{\dagger}$ \\
REST & $0.29 \pm 0.003$ & $0.86 \pm 0.01$ & $0.338 \pm 0.001$ & $0.019 \pm 0.001$
\end{tabular}

$515 \overbrace{}^{\dagger}$ Indicates significantly different from REST. Data are mean \pm SD.

516

517

518

519

520

521

522

523

524

525

526

527

528

529

530

531

532

533 
534 Figure 1. A) Energy intake (kJ) at each meal; B) Total and relative energy intake (kJ) for EX ( $535 \square)$ and REST ( $\square$ ) trials. ${ }^{\dagger}$ Indicates significantly different from REST trial. Data are mean \pm 536 SD.

A)

$\square$ EX $\square$ REST

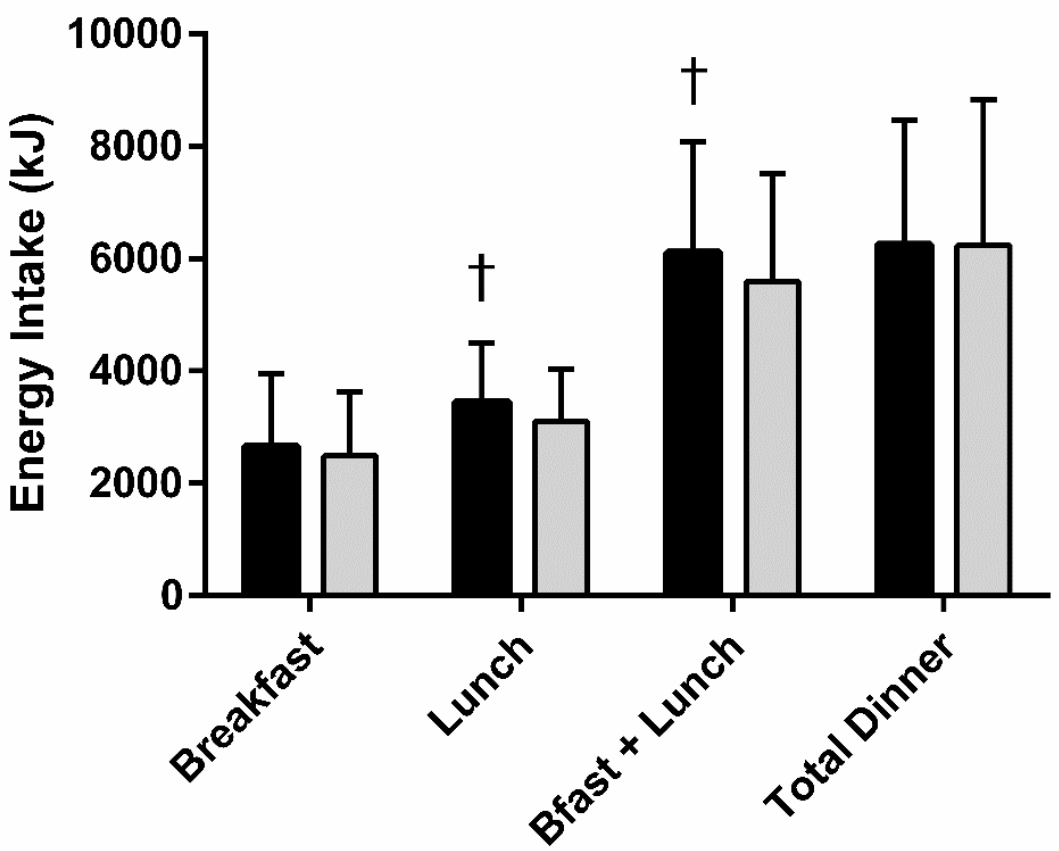

B)

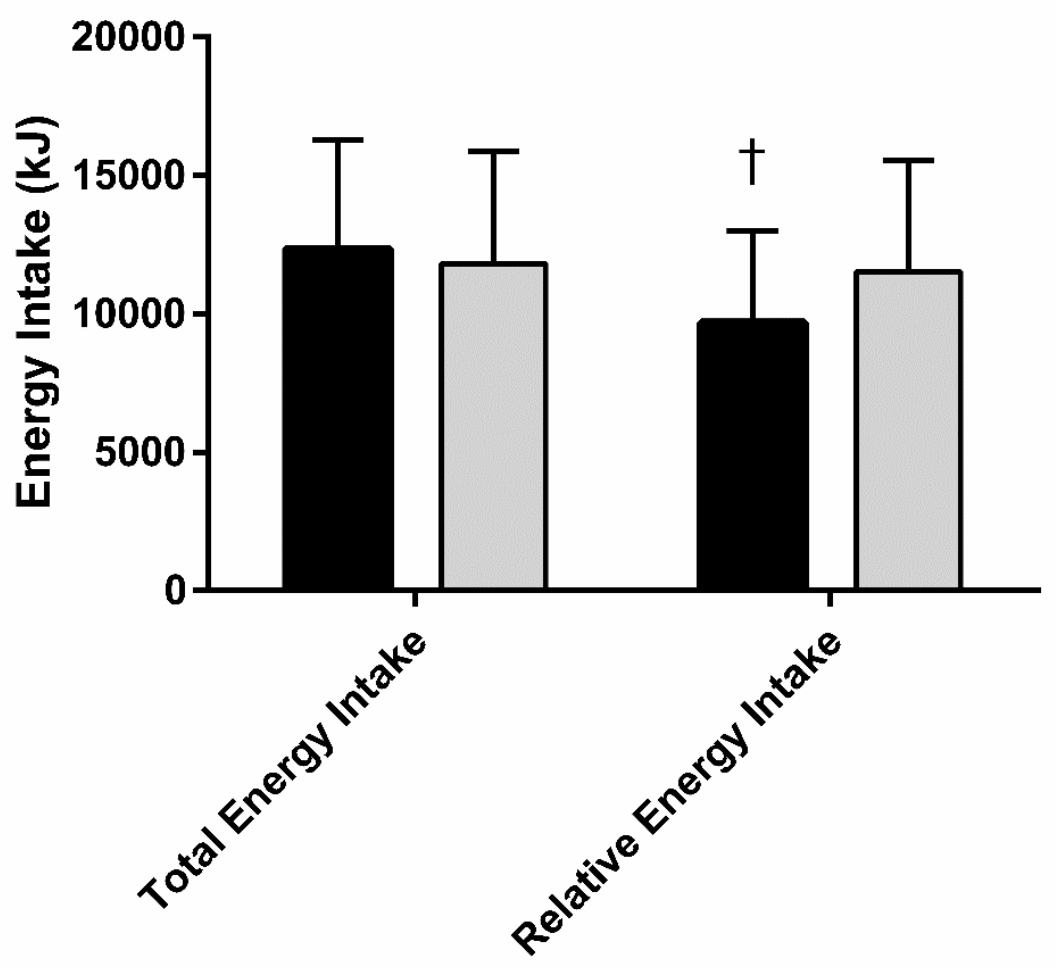


538 Figure 2. Change in A) Hunger, B) Fullness, C) Desire to eat (DTE) and D) Prospective food 539 consumption (PFC) over the trial day for both EX $(-\rightarrow)$ and REST (-). ${ }^{\dagger}$ Indicates significantly 540 different from REST trial. Data are mean \pm SEM.

A)

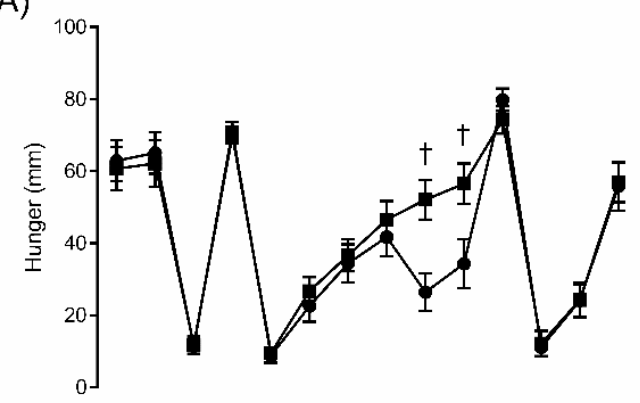

C)

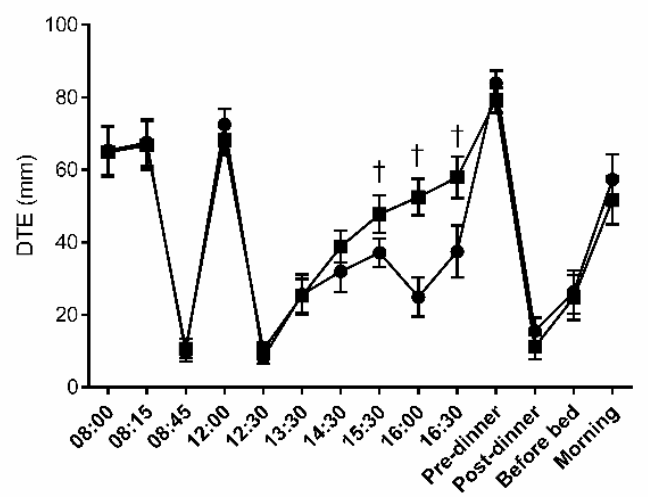

B)

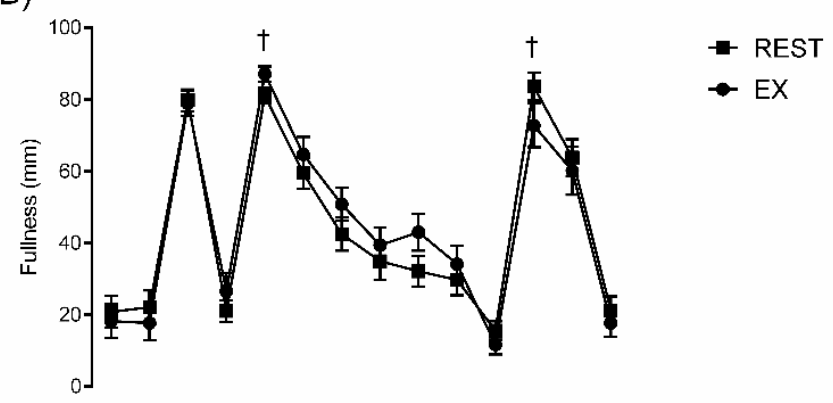

D)

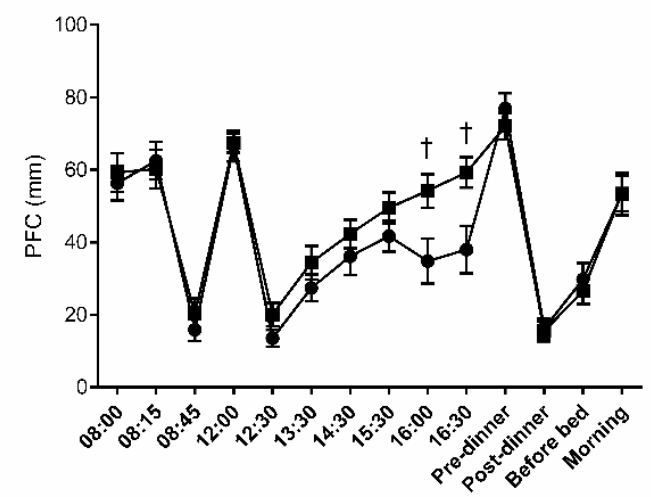

541 